\title{
Mobile male-killer: similar Wolbachia strains kill males of divergent Drosophila hosts
}

\author{
SL Sheeley and BF McAllister \\ Department of Biology and Roy J Carver Center for Comparative Genomics, University of Iowa, Iowa City, IA, USA
}

\begin{abstract}
Wolbachia are capable of eliciting a variety of reproductive phenotypes from their hosts, including the production of an all-female progeny through embryonic male-killing. To date, phylogenetic analyses indicate six independent acquisitions of the ability to kill male embryos among Wolbachia strains which infect insects. Of these six strains, only one appears to have experienced horizontal transmission between host species while maintaining a male-killing phenotype. The rarity of male-killing Wolbachia and their disjunct phylogenetic relationships is surprising, given the apparently common occurrence of horizontal transfer involving Wolbachia strains causing other phenotypes. A male-killing Wolbachia strain examined here in Drosophila borealis
\end{abstract}

represents a second case of apparent horizontal transmission, based on its close relationship to a male-killing strain in a distantly related Drosophila species. The results reported here show that this Wolbachia has maintained a stable phenotype in $D$. borealis over a period of at least 50 years, and that a similar strain elicits the same male-killing phenotype in a second Drosophila species, indicating that male-killing may be a stable long-term strategy. Sampling bias and/or a lack of suitable hosts are discussed as possible causes of the low frequency of male-killers identified among Wolbachia strains.

Heredity (2009) 102, 286-292; doi:10.1038/hdy.2008.126; published online 14 January 2009

Keywords: Drosophila borealis; horizontal transmission; reproductive parasitism

\section{Introduction}

Insects are hosts to a diverse assemblage of microorganisms, some of which are able to manipulate host reproduction to their own advantage. Perhaps the best studied of these reproductive parasites is the $\alpha$-proteobacteria Wolbachia. Wolbachia inhabit $\sim 66 \%$ of insect species, as well as some isopods and nematodes (Hilgenboecker et al., 2008). They cause reproductive phenotypes such as cytoplasmic incompatibility between infected and uninfected hosts, feminization of genetic males, parthenogenesis and embryonic male-killing (Stouthamer et al., 1999). In each of these cases, manipulation of host reproduction increases the fitness of the maternally transmitted microbe. The evolutionary history of Wolbachia is characterized by rampant horizontal transmission among hosts and repeated phenotypic shifts among cytoplasmic incompatabilityinducing, feminizing, parthenogenesis-inducing and male-killing strains. Male-killing Wolbachia strains are observed only rarely, and this phenotype appears to be independently derived in most cases. The rare origin of this unique phenotype may reflect the instability of the male-killing phenotype with strains frequently changing to a different phenotype, an inability of male-killing lineages to successfully sustain infection in novel hosts

Correspondence: SL Sheeley, Department of Biology, University of Iowa, Iowa City, IA 52242-1324, USA

E-mail: sara-sheeley@uiowa.edu

Received 3 June 2008; revised 27 October 2008; accepted 3 December 2008; published online 14 January 2009 resulting in strain extinction, or a host-dependence with few host species eliciting a male-killing response.

Among the Wolbachia phenotype classes, male-killers impose the greatest fitness cost on their hosts due to the embryonic lethality of all male offspring (Charlat et al., 2003). Selection favors the suppression of male-killing caused by the bacteria, but evidence for suppressors has been observed in only one male-killing infection (Hornett et al., 2006). Theoretically, it is suggested that the maintenance of an equilibrium frequency of infection by a male-killer is possible only if there is an increase in fitness experienced by the female offspring of infected female hosts. Benefits of having an infected mother are attributed to reductions in sibling competition, inbreeding and egg cannibalism (Hurst, 1991). In addition, parasite characteristics, such as transmission efficiency, affect the level of infection within a host population and are influenced by abiotic factors such as temperature. All of these parameters must reach a delicate balance to maintain an infection without parasite fixation, resulting in complete loss of males and extinction of both the host and the endosymbiont.

Although horizontal transmission of Wolbachia is common, evidence of horizontal transmission of a male-killing strain of Wolbachia has been found in only one case of two distantly related butterfly hosts (Dyson et al., 2002). The presence of additional clades of malekilling Wolbachia would indicate that male-killing can be maintained over long time periods and among different hosts, and that the lack of observed horizontal transmission may be because of both the scarcity of appropriate hosts and the biased sampling of male-killing strains. To date, only two strains of male-killing Wolbachia have 
been identified within the genus most widely used as an insect genetic model, Drosophila, and phylogenetic evidence shows that the strains have independent origins (Dyer and Jaenike, 2004). Incidences of femalebiased sex ratios have been reported in several other Drosophila species that have yet to be attributed to a specific causal agent. One factor influencing the observed rarity of male-killing Wolbachia among Drosophila is the inherent difficulty of discovery. Lines that are host to male-killing strains would likely be lost with normal maintenance, so stock center screens to identify the presence of bacterial symbionts, including Wolbachia, fail to identify male-killing strains (Mateos et al., 2006).

Carson (1956) originally described a line of Drosophila borealis that produced a female-biased sex ratio. A recent isolate of this species also produced an all-female progeny in laboratory culture and preliminary screening of this line revealed the presence of Wolbachia. Our characterization of this line shows this to be a third malekilling Wolbachia infection within the genus Drosophila. Analysis of ovarian bacterial flora shows almost exclusively Wolbachia, and the presence of that infection is associated with a $50 \%$ reduction in egg hatch and the loss of male progeny. Phylogenetic analysis of DNA sequences of wolbachia surface protein (wsp) and multilocus sequence typing (MLST) system genes shows that the male-killing strain in $D$. borealis forms a common clade with the male-killing Wolbachia strain infecting Drosophila innubila. The presence of closely related strains of malekilling Wolbachia in two distantly related hosts suggests the maintenance of the male-killing phenotype through at least one incidence of horizontal transmission.

\section{Materials and methods}

A line of $D$. borealis was established with the offspring of a single female collected near Oshkosh, Wisconsin $\left(44^{\circ} 6.13^{\prime} \mathrm{N}, 88^{\circ} 55.26^{\prime} \mathrm{W}\right)$. This female produced $100 \%$ female offspring. The line was maintained in the laboratory by outcrossing with males from another line collected at the same locality. Approximately nine generations of outcrossing were performed before this study, thus homogenizing the nuclear genetic background in the two lines.

\section{Survey of ovarian bacterial flora}

The causal relationship between the presence of Wolbachia and the sex ratio trait was investigated by identifying microorganisms in the ovaries of affected flies, followed by antibiotic curing of the infection. Ovarian DNA was isolated from sexually mature, virgin female flies from the all-female and normal sex ratio lines, and earlier published general primers were used to amplify eubacterial ribosomal DNA (Weisburg et al., 1991). Amplified DNA was cloned into a TOPO TA vector (Invitrogen, Carlsbad, CA, USA), and clones were sequenced. The resulting sequences were used to design a restriction digestion assay specific for the Wolbachia strain present, and additional clones were typed.

\section{Antibiotic curing of sex ratio trait}

Female flies from each line were crossed with males from the normal sex ratio line and allowed to oviposit either on standard cornmeal media or on media containing $0.025 \%$ tetracycline. The resulting female offsprings were crossed twice more to normal males, and these two generations (egg to adult) were also reared on each food type. After each generation, offspring were sexed and counted. DNA was extracted from the ovaries of treated and untreated females from each line and Wolbachiaspecific primers for the wsp gene were used to determine the Wolbachia infection status. Third generation offsprings from the tetracycline-treated all-female line were used to establish a cured line derived from the all-female line.

\section{Analysis of egg viability}

In order to differentiate between feminization and male-killing as the cause of the sex ratio distortion, a comparison of egg viability was carried out among allfemale, normal and cured flies. Female flies from each line were mated en masse to normal males in population cages and allowed to oviposit on grape-juice agar supplemented with live yeast. Eggs were collected twice daily from each line and arrayed on fresh grape-juice agar plates. After $48 \mathrm{~h}$, the proportion of eggs hatched was assayed for each collection by visually inspecting each egg. Egg hatch data were analyzed by carrying out a logistic regression to test the effects of line on egg hatch proportion while controlling for effects of the blocked design created by collecting eggs over several days (PROC GLIMMIX, SAS v9.1). Egg hatch proportion from the cured line was compared pairwise to that of the all-female and normal sex ratio lines.

\section{Phylogenetic analysis of Wolbachia sex ratio distorters}

To determine the phylogenetic position of the Wolbachia strain in $D$. borealis, portions of the wsp and the Wolbachia MLST genes (ftsZ, $\operatorname{cox} A, \operatorname{gat} B, h c p A$ and $f b p A$ ) were amplified from ovarian DNA of the all-female line using earlier published primer sequences (Zhou et al., 1998; Baldo et al., 2006). Sequences of the amplified products were determined using routine methods, and reference sequences from other strains were retrieved from GenBank. Accession numbers for sequences from other strains are available as supplementary material. Sequences were aligned manually. All parsimony analyses were carried out in PAUP*4.0b10 (Swofford, 2002). The model selection for Bayesian analysis was carried out using Akaike Information Criterion as implemented in Modeltest 3.6 (Posada and Crandall, 1998). Bayesian posterior probabilities were generated using $\mathrm{Mr}$ Bayes v3.1.2 (Huelsenbeck and Ronquist, 2001; Ronquist and Huelsenbeck, 2003).

Wolbachia in insects belong to two deeply divergent clades. Sequences of wsp were used to determine the phylogenetic position of the strain infecting $D$. borealis relative to earlier described strains. This is the fastest evolving protein known in Wolbachia, and has been used extensively for phylogenetic analysis (Zhou et al., 1998). Sequences from both insect-hosted clades were included, and sequences of two strains from a distantly-related clade from nematode hosts were used to root the phylogeny. All known male-killing strains were included in the analysis. Parsimony criteria were used to reconstruct a phylogeny with $511 \mathrm{bp}$ of sequence from 39 strains of Wolbachia using the default heuristic settings in PAUP* 4.0b10 (Swofford, 2002). Confidence in nodes was determined by 10000 bootstrap replicates of the 
maximum parsimony analysis and by Bayesian posterior probabilities using a GTR $+\Gamma+\mathrm{I}$ model. Posterior probabilities were generated from 300000 Markov chain Monte Carlo generations with 75000 discarded as burn-in.

A more robust analysis of the phylogenetic position of the strain in $D$. borealis relative to highly similar strains was conducted using wsp (460 bp) and MLST genes: ftsZ (435 bp), gatB (368 bp), coxA (403 bp), hcpA (444bp) and $f b p A(428 \mathrm{bp})$. An exhaustive parsimony search was performed. Confidence in nodes was determined by 10000 bootstrap replicates of the maximum parsimony analysis and by Bayesian posterior probabilities using a GTR model. Posterior probabilities were generated from 200000 Markov chain Monte Carlo generations with 50000 discarded as burn-in.

An MLST system has been developed specifically for Wolbachia as a complement to phylogenetic analysis (Baldo et al., 2006). The MLST system consists of a database of allelic profiles of five slowly evolving loci. Each distinct allele at each locus is given a numeric designation, so that each strain has a five-number allelic profile, and each distinct allelic profile is designated as a unique sequence type. Only sequences that are identical for a given gene will share the same number in their allelic profile for that locus, and only strains whose sequence is identical at all five loci will share the same sequence type. Strains with identical alleles of 3 or more of the five MLST loci share a sequence-type complex. In addition, a second database catalogs the amino-acid sequences of four hyper-variable regions of wsp. This allows the detection of allele shuffling by recombination and comparison of the congruence of wsp and the MLST loci. Searches on the MLST database were conducted through the website (http://pubmlst.org/wolbachia/) developed by Jolley et al. (2004).

Inference about the origins of infection among hosts relies on a comparison of their phylogenetic relationships in conjunction with those of the endosymbionts. Given the inferred relationships among the major species group in the genus Drosophila, classification of $D$. borealis and $D$. innubila in the virilis and quinaria species groups, respectively, indicates a distant relationship between these host species. Specific resolution of their phylogenetic relationship was obtained with available sequence data analyzed within the framework of the reference genome sequences of Drosophila species. Sequences of mitochondrial genes COI and COII of D. borealis, $D$. innubila and D. recens, and two and three regions of nuclear genes of $D$. recens and $D$. innubila, respectively, were aligned with the corresponding regions from the reference genome sequences of nine Drosophila species. Over $20 \mathrm{~kb}$ of additional sequence from nine reference species contained 14 genes that yielded method-independent inference of the consensus topology among 12 species of Drosophila in the analysis of Rasmussen and Kellis (2007). A table listing the data included in this supermatrix is available as supplementary material. Overall tree topology and branch lengths were obtained using Maximum Likelihood in PAUP under the GTR $+\Gamma+$ I model, with posterior probabilities for each node estimated in Mr Bayes.

\section{Results}

\section{Survey of ovarian bacterial flora}

A Wolbachia infection causing this sex ratio trait would be expected to be prevalent in the ovaries of affected females. Preliminary sequencing of eight bacterial ribosomal DNA clones from the ovarian DNA of the all-female line revealed mostly Wolbachia sequences (seven of eight sequences). The single unique sequence showed significant BLAST to uncultured bacterial strains. The presence of this bacterium was attributed to contamination from gut flora during ovary dissection. A restriction digest assay was designed to differentiate the sequence of the Wolbachia strain from the ribosomal DNA of a variety of other microorganisms, including the uncultured bacterium and all known types of reproductive parasites. Of the 54 additional clones screened, all tested positive as Wolbachia. All attempts to amplify bacterial DNA failed with ovarian DNA from the normal sex ratio line.

\section{Antibiotic curing of sex ratio trait}

Further correlation of the sex ratio trait with Wolbachia infection was provided by their simultaneous loss after treatment with antibiotics. After rearing flies for a single generation on a medium containing tetracycline, the normal sex ratio line remained $\sim 50 \%$ male, whereas the all-female line produced no males. The all-female line responded to tetracycline treatment after a second generation of culture in the presence of tetracycline, producing an approximately $1: 1$ sex ratio. This sex ratio remained stable after a third generation of antibiotic treatment. Non-tetracycline-treated controls continued to produce only female offspring and sex ratio remained at 1:1 in the normal sex ratio line regardless of treatment for the duration of the experiment (Table 1). Failure to amplify Wolbachia DNA corresponded with the loss of the sex ratio trait.

\section{Analysis of egg viability and $Y$ chromosome inheritance} The mode of male loss was ascertained by comparing the egg hatch of the all-female line with that of controls. Male-killing will result in a 50\% reduction in hatch, assuming a 1:1 primary sex ratio. Eight collections of 160 eggs per line were made (1280 eggs/line). Egg hatch proportions for normal and cured lines were $0.77 \pm 0.02$

Table 1 Sex ratio (male/female) of offspring after tetracycline treatment

\begin{tabular}{|c|c|c|c|c|c|c|}
\hline \multirow[t]{2}{*}{ Tetracycline } & \multicolumn{2}{|c|}{ Generation 1} & \multicolumn{2}{|c|}{ Generation 2} & \multicolumn{2}{|c|}{ Generation 3} \\
\hline & - & + & - & + & - & + \\
\hline Normal sex ratio & $0.48 \pm 0.03$ & $0.54 \pm 0.03$ & $0.53 \pm 0.07$ & $0.52 \pm 0.04$ & $0.46 \pm 0.05$ & $0.51 \pm 0.04$ \\
\hline All female & $0.00 \pm 0.00$ & $0.00 \pm 0.00$ & $0.00 \pm 0.00$ & $0.53 \pm 0.12$ & $0.00 \pm 0.00$ & $0.50 \pm 0.04$ \\
\hline
\end{tabular}




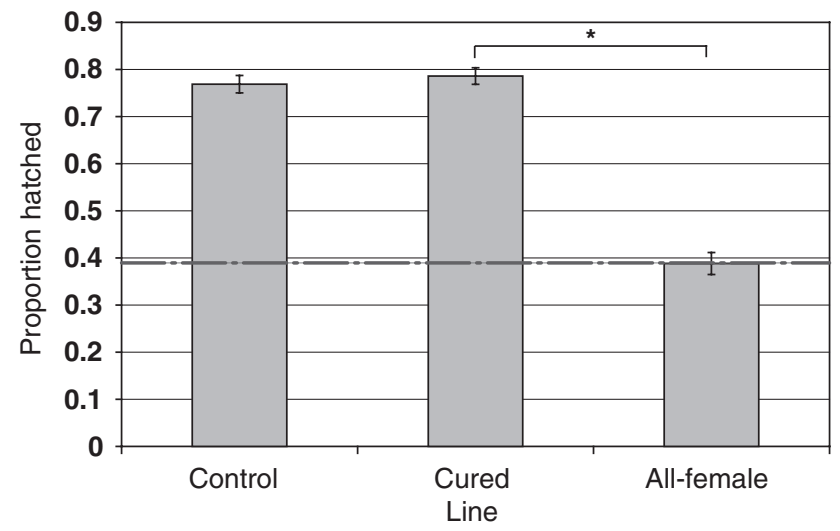

Figure $1 \mathrm{Egg}$ hatch proportion for control, cured and all-female lines. Dashed line indicates $50 \%$ of the average hatch of the control and cured lines. ${ }^{*} P<0.0001$

and $0.78 \pm 0.02$, respectively, whereas egg hatch proportion for the all-female line was $0.38 \pm 0.02$ (Figure 1). A logistic regression was fitted to the data and showed a significant effect of line on egg hatch $(P<0.0001)$. Post-hoc pair-wise comparisons showed a significant difference between hatch proportion of all-female and cured lines $(P<0.0001)$, but not between normal and cured lines. The approximately $50 \%$ reduction in egg hatch in the allfemale line is indicative of specific lethality of males. Further indication of male lethality is provided by the failure to amplify the Y-chromosome gene $K L-2$ from DNA of the all-female line as well as DNA from females of the normal sex ratio line. Amplification was successful with DNA from males of the normal sex ratio line (data not shown).

\section{Phylogenetic analysis of Wolbachia sex ratio distorters} Horizontal transmission of Wolbachia is evidenced by the presence of identical or similar strains in divergent hosts. Phylogenetic incongruence between hosts and endosymbionts indicates independent infection of the two hosts by the same strain, rather than inheritance from a common ancestor. Within $511 \mathrm{bp}$ of aligned wsp sequence, only two nucleotide differences are present between the male-killing Wolbachia strains infecting $D$. borealis and $D$. innubila. The most parsimonious reconstruction showed the $D$. borealis strain as sister to the male-killing strain of Wolbachia infecting D. innubila, but owing to the minimal divergence in these short sequences, bootstrap support for the node connecting the $D$. borealis and D. innubila strains is low (Figure 2). The two male-killers grouped with five strains exhibiting minimal divergence, and mostly present among several divergent clades of Drosophila. Consistent with earlier results (Dyer and Jaenike, 2004), the third male-killing strain of Wolbachia in D. bifasciata grouped separately from that of $D$. borealis and $D$. innubila. The previously recognized ' $\mathrm{A}$ ', ' $\mathrm{B}$ ' and ' $\mathrm{C}$ ' clades of Wolbachia were resolved with high confidence.

The $D$. borealis male-killer and five other closely related strains were examined in a second phylogenetic analysis. The genes used in this analysis were chosen because they are part of the MLST system for Wolbachia, and sequences are available for many strains (Baldo et al., 2006).
Typically, the MLST genes are not combined with wsp sequences in phylogenetic analyses because wsp is known to recombine frequently among strains (Baldo et al., 2005; Baldo and Werren, 2007), leading to incongruence in combined datasets. The strains investigated here, though, are so similar that their relationships have not been influenced by recombination at wsp. A partition homogeneity test confirmed that the genes show no incongruence, both among all genes, and between $w s p$ and the five MLST genes $(P=1.0)$. Although the taxa for this analysis were chosen on the basis of their similarity to the male-killing strain at wsp, those strains are also the most closely related from the MLST database, confirming that within this group, ws $p$ and the MLST genes share a similar evolutionary history. This analysis consisted of 2539 characters and confirmed the sister relationship between the $D$. borealis and $D$. innubila male-killers with strong support for a common node (Figure 3a). Approximately $3500 \mathrm{bp}$ of sequence was obtained in total from the newly-characterized male-killing strain, and all sequences, except that of $w s p$, is identical in the two male-killing strains, including ftsZ (614 bp), gatB (368 bp), cox $A$ (403 bp), hcp $A$ (444bp), fbpA (428 bp) and 16s rDNA (694bp).

The male-killing strains infecting $D$. borealis and $D$. innubila have the same MLST allelic profile, and therefore the same sequence type (10) and sequence-type complex (STC-13), but differ by one nonsynonymous change in two of the four hypervariable regions of wsp. The MLST database includes another strain (id 83) with an identical MLST allelic profile that only differs from the strain infecting $D$. borealis by a single nonsynonymous change in one hypervariable region (Table 2). This strain, identified in Drosophila munda, was amplified from a single specimen of unknown sex, and has an unknown effect on the host species (Julie Stahlhut, personal communication). This strain was not included in our phylogenetic analysis, as we cannot draw any conclusions with regard to the evolutionary history of the malekilling phenotype without additional information. Other strains with phenotypic information in the database are more distantly related to the clade of male-killing strains shared between $D$. borealis and $D$. innubila.

The two host species, $D$. borealis and $D$. innubila, belong to widely divergent species groups, the virilis group and the quinaria group, respectively. Phylogenetic analysis of these species with ten additional Drosophila species, nine of which have complete genome sequences, confirmed that $D$. borealis and $D$. innubila are deeply divergent and belong to lineages that diverged before the colonization of the Hawaiian archipelago by Drosophila (Figure 3b), an event estimated to have occurred 25-42 mya (Desalle, 1992; Powell and Desalle, 1995; Tamura et al., 2004).

\section{Discussion}

Females of the all-female line harbor almost exclusively Wolbachia in their ovaries, and the infection and sex ratio trait are eliminated on antibiotic treatment. Since the conclusion of this experiment, the cured line has been maintained over 20 generations, and the sex ratio remains at 50:50. Egg viability is reduced by 50\% in the all-female line compared with control and cured lines, consistent with complete lethality of male embryos, and there is no evidence of feminization of genetic males as 


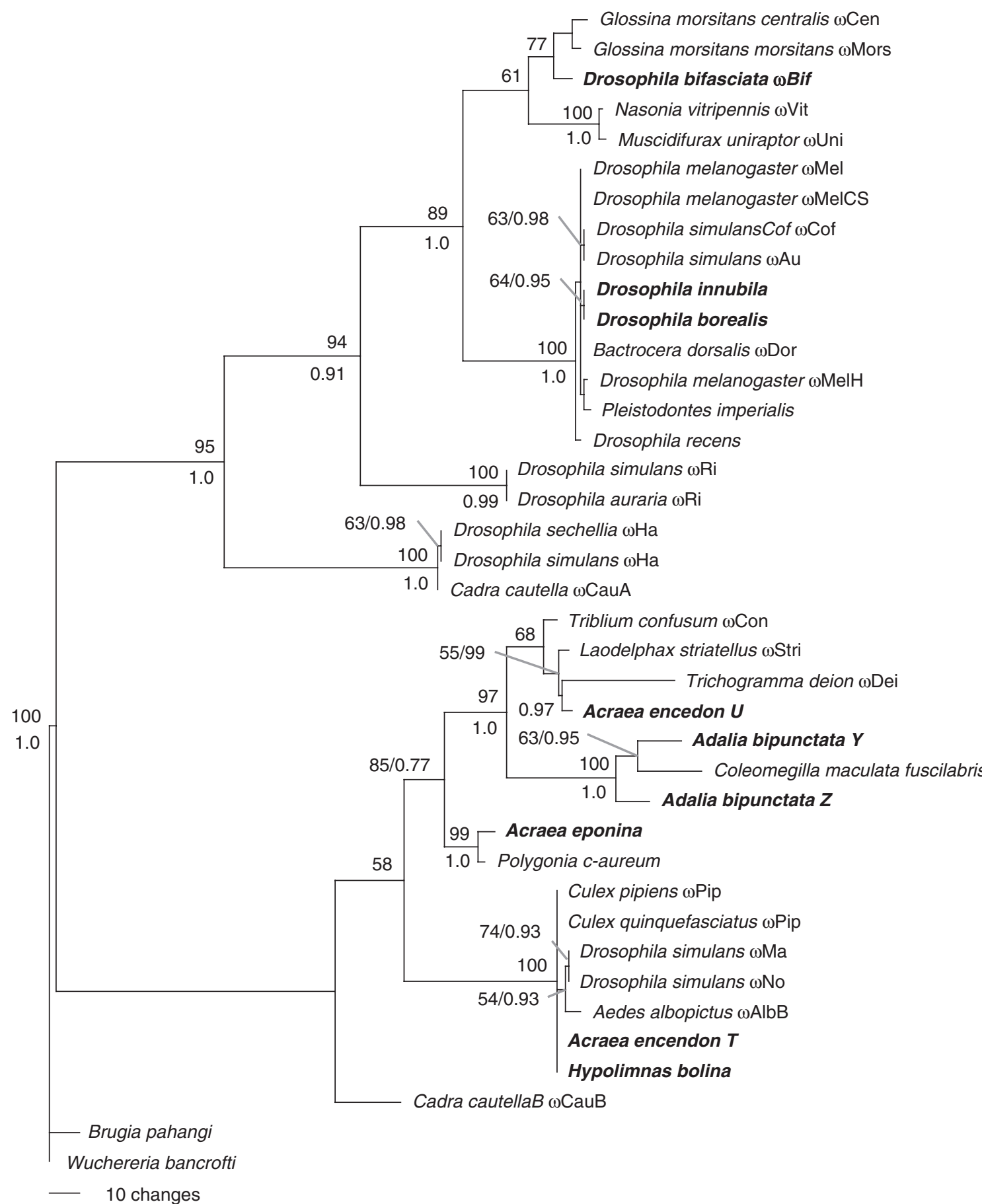

Figure 2 Most parsimonious reconstruction of 39 Wolbachia wsp sequences. Bootstrap values are shown for 10000 parsimony replicates (top) as well as Bayesian posterior probabilities (bottom). Male-killing lineages are shown in bold, and the major divisions of the Wolbachia clades are shown as ' $\mathrm{A}$ ', ' $\mathrm{B}$ ' and ' $\mathrm{C}$ '.

investigated by the attempted amplification of a Y-linked gene. Together, these results indicate that the Wolbachia strain infecting $D$. borealis is an embryonic male-killer.

Phylogenetic analysis shows that this strain of malekilling Wolbachia is very closely related to another malekilling strain infecting D. innubila, a species that shared a common ancestor with $D$. borealis 25-42 mya. This close relationship between the parasites of two distantly related hosts is a hallmark of horizontal transmission, and may reflect a common clade of Wolbachia that has established and maintained a male-killing phenotype in more than one host. Although the available data indicate a similar strain of Wolbachia that causes male-killing in these divergent hosts, insufficient sampling and characterization of phenotypic effects in host species infected with this Wolbachia clade may generate a spurious association between these male-killing strains. Switching between phenotypes may occur on such a rapid timescale that male-killing in $D$. borealis and D. innubila results from convergence, rather than it being the 

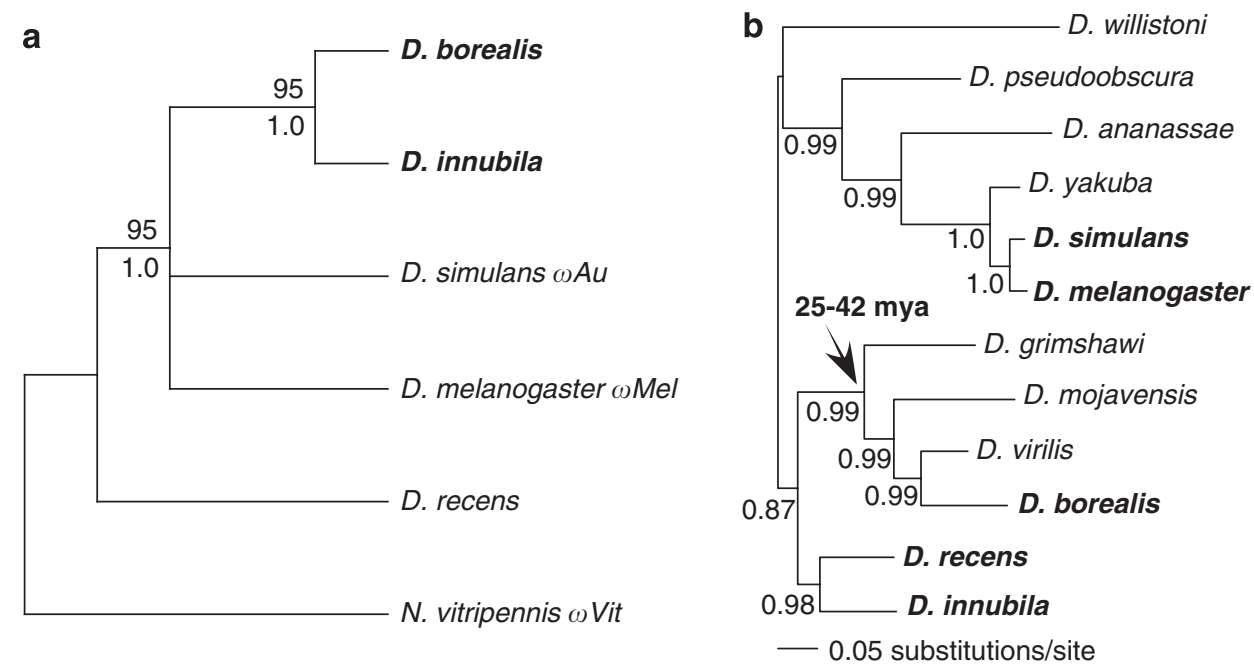

Figure 3 (a) Most parsimonious reconstruction of sequences representing the Wolbachia strains most closely related to the D. borealis malekilling strain. For each strain, partial sequences from five genes were used: wsp (460 bp), ftsZ (435 bp), gatB (368 bp), coxA (403 bp), hcp $A$ $(444 \mathrm{bp})$ and $f b p A(429 \mathrm{bp})$. Bootstrap values for 10000 parsimony replicates (top) and Bayesian posterior probabilities (bottom) are shown. Male-killing lineages are shown in bold. (b) Phylogenetic reconstruction of representative Drosophila species. Those species that are host to Wolbachia strains included in ' $a$ ' are shown in bold. Bayesian posterior probabilities are indicated at each node.

Table $2 \mathrm{Wsp}$ and MLST allelic profiles of D. borealis and the two most closely related isolates from the Wolbachia MLST database

\begin{tabular}{|c|c|c|c|c|c|c|c|c|c|c|c|}
\hline \multirow[b]{2}{*}{ Host } & \multicolumn{4}{|c|}{ Wsp HVR } & \multicolumn{5}{|c|}{$M L S T$} & \multirow[b]{2}{*}{$S T$} & \multirow[b]{2}{*}{$S T-C$} \\
\hline & 1 & 2 & 3 & 4 & gatB & $\operatorname{cox} A$ & hсpA & ftsZ & $f b p A$ & & \\
\hline & 1 & Unique & 13 & 70 & 1 & 1 & 1 & 3 & 2 & 10 & 13 \\
\hline D. munda & 1 & 10 & 13 & 70 & 1 & 1 & 1 & 3 & 2 & 10 & 13 \\
\hline D. innubila & 1 & 10 & 13 & 10 & 1 & 1 & 1 & 3 & 2 & 10 & 13 \\
\hline
\end{tabular}

Abbreviations: HVR, hypervariable region; MLST, multilocus sequence typing; ST, sequence type; ST-C, sequence type complex.

ancestral mode of transmission of this Wolbachia clade. The clade also infects $D$. munda, but the absence of phenotypic analysis of the infection does not help resolve the persistence of the male-killing phenotype. Further sampling coupled with phenotypic analysis is needed to determine if male-killing is ancestral to this clade, and whether other phenotypes arise as a consequence of host suppression. This has been observed among populations of a single host species of another male-killer, infecting Hypolimnas bolina. In this case, suppression of malekilling in some populations results in the expression of a cytoplasmic incompatibility phenotype (Hornett et al., 2008).

Interestingly, the two distantly related hosts of malekilling strains, $D$. borealis and D. innubila, do not share any portion of their natural ranges. $D$. borealis is found in the Great Lakes region of North America, whereas $D$. innubila is found solely on mountains known as sky islands in the deserts of the Southwest USA and Mexico. Parasitoid wasps are capable of transferring Wolbachia between distantly-related host species (Heath et al., 1999), and parasitoids often harbor Wolbachia infections that appear closely related to those of their hosts at the wsp locus (Vavre et al., 1999), but our limited knowledge of the ecology of these species precludes identification of the intermediate transmission vector. Gaps in infection over geographic and phylogenetic space suggest that only certain species may be adequate for sustained infection.

This result, coupled with the presence of a male-killing clade in butterflies (Dyson et al., 2002), indicates that malekilling may be a sustainable strategy in some Wolbachia clades and that the male-killing phenotype can be maintained during transmission among different hosts. This has been investigated earlier at the intraspecific level, in which experimental transfer of the male-killing Wolbachia infecting Drosophila bifasciata into lines established from historically uninfected populations resulted in successful embryonic killing (Veneti et al., 2004). Recent analysis of another male-killing microorganism, Spiroplasma, indicates a reduction in male-killing efficiency with increased divergence between the original and novel hosts (Tinsley and Majerus, 2007). Although the biology of Spiroplasma and Wolbachia male-killers may be very different, the authors observed highly successful malekilling after intragenus transfer, consistent with our finding that this strain of Wolbachia is a highly efficient male-killer in both $D$. borealis and $D$. innubila. This malekilling strain appears to be capable of maintaining a malekilling phenotype among hosts and, for some time, within hosts (evidence from $D$. borealis indicates at least 50 years), so sampling bias owing to the lack of suitable hosts may explain the rarely discovered examples of male-killing Wolbachia, rather than an inherent instability of the phenotype. Characterization of a third male-killing Wolbachia infection in Drosophila will allow investigation of requirements for sustained infection, and allow powerful interspecific comparisons for investigating the mechanism of male-killing. The ability of this strain to selectively kill male embryos in two divergent species indicates that it may be possible for it to successfully infect and kill male embryos of D. melanogaster, potentially unleashing its extensive genetic toolkit to identify the mechanism of male killing. 


\section{References}

Baldo L, Dunning Hotopp JC, Jolley KA, Bordenstein SR, Biber SA, Choudhury RR et al. (2006). Multilocus sequence typing system for the endosymbiont Wolbachia pipientis. Appl Environ Microbiol 72: 7098-7110.

Baldo L, Lo N, Werren JH (2005). Mosaic nature of the Wolbachia surface protein. J Bacteriol 187: 5406-5418.

Baldo L, Werren JH (2007). Revisiting Wolbachia supergroup typing based on WSP: spurious lineages and discordance with MLST. Curr Microbiol 55: 81-87.

Carson HL (1956). A female-producing strain of D. borealis Patterson. 30: 109-110.

Charlat S, Hurst GD, Mercot H (2003). Evolutionary consequences of Wolbachia infections. Trends Genet 19: 217-223.

Desalle R (1992). The origin and possible time of divergence of the Hawaiian Drosophilidae-evidence from DNA-sequences. Mol Biol Evol 9: 905-916.

Dyer KA, Jaenike J (2004). Evolutionarily stable infection by a male-killing endosymbiont in Drosophila innubila: molecular evidence from the host and parasite genomes. Genetics 168: 1443-1455.

Dyson EA, Kamath MK, Hurst GD (2002). Wolbachia infection associated with all-female broods in Hypolimnas bolina (Lepidoptera: Nymphalidae): evidence for horizontal transmission of a butterfly male killer. Heredity 88: 166-171.

Heath BD, Butcher RD, Whitfield WG, Hubbard SF (1999). Horizontal transfer of Wolbachia between phylogenetically distant insect species by a naturally occurring mechanism. Curr Biol 9: 313-316.

Hilgenboecker K, Hammerstein P, Schlattmann P, Telschow A, Werren JH (2008). How many species are infected with Wolbachia?-A statistical analysis of current data. FEMS Microbiol Lett 281: 215-220.

Hornett EA, Charlat S, Duplouy AM, Davies N, Roderick GK, Wedell $\mathrm{N}$ et al. (2006). Evolution of male-killer suppression in a natural population. PLoS Biol 4: e283.

Hornett EA, Duplouy AM, Davies N, Roderick GK, Wedell N, Hurst GD et al. (2008). You can't keep a good parasite down: evolution of a male-killer suppressor uncovers cytoplasmic incompatibility. Evolution 62: 1258-1263.

Huelsenbeck JP, Ronquist F (2001). MRBAYES: Bayesian inference of phylogenetic trees. Bioinformatics 17: 754-755.

Hurst LD (1991). The incidences and evolution of cytoplasmic male killers. Proc R Soc Lond B 244: 91-99.
Jolley KA, Chan M, Maiden MCJ (2004). mlstdbNet-distributed multi-locus sequence typing (MLST) databases. BMC Bioinformatics 5: 86.

Mateos M, Castrezana SJ, Nankivell BJ, Estes AM, Markow TA, Moran NA (2006). Heritable endosymbionts of Drosophila. Genetics 174: 363-376.

Posada D, Crandall KA (1998). MODELTEST: testing the model of DNA substitution. Bioinformatics 14: 817-818.

Powell JR, Desalle R (1995). Drosophila molecular phylogenies and their uses. In: Hecht MK, MacIntyre RJ, Clegg MT (eds) Evolutionary Biology, vol. 28. Plenum Press: New York, pp 87-138.

Rasmussen MD, Kellis M (2007). Accurate gene-tree reconstruction by learning gene- and species-specific substitution rates across multiple complete genomes. Genome Res 17: 1932-1942.

Ronquist F, Huelsenbeck JP (2003). MrBayes 3: Bayesian phylogenetic inference under mixed models. Bioinformatics 19: 1572-1574.

Stouthamer R, Breeuwer JA, Hurst GD (1999). Wolbachia pipientis: microbial manipulator of arthropod reproduction. Annu Rev Microbiol 53: 71-102.

Swofford DL (2002). PAUP* Phylogenetic Analysis Using Parsimony ( ${ }^{*}$ and Other Methods). Version 4 Sinaur Associates: Sunderland, MA.

Tamura K, Subramanian S, Kumar S (2004). Temporal patterns of fruit fly (Drosophila) evolution revealed by mutation clocks. Mol Biol Evol 21: 36-44.

Tinsley MC, Majerus ME (2007). Small steps or giant leaps for male-killers? Phylogenetic constraints to male-killer host shifts. BMC Evol Biol 7: 238.

Vavre F, Fleury F, Lepetit D, Fouillet P, Bouletreau M (1999). Phylogenetic evidence for horizontal transmission of Wolbachia in host-parasitoid associations. Mol Biol Evol 16: 1711-1723.

Veneti Z, Toda MJ, Hurst GD (2004). Host resistance does not explain variation in incidence of male-killing bacteria in Drosophila bifasciata. BMC Evol Biol 4: 52.

Weisburg WG, Barns SM, Pelletier DA, Lane DJ (1991). 16S ribosomal DNA amplification for phylogenetic study. J Bacteriol 173: 697-703.

Zhou W, Rousset F, O'Neil S (1998). Phylogeny and PCR-based classification of Wolbachia strains using wsp gene sequences. Proc Biol Sci 265: 509-515. 